\title{
Der Varix aneurysmaticus und die Kasuistik des Königlich Württembergischen Regimentsarztes Dr. Seeger anno 1825
}

Der Londoner Wundarzt William Hunter (1718-1783) hat schon anno 1764 festgestellt, dass sich beim Aneurysma verschiedene Formen unterscheiden lassen [1, 8, 18] (Box 1). Das Aneurysma verum war in diesen Zeiten als Spätsymptom der Syphilis häufig an der Aorta zu beobachten und konnte erhebliche Ausmaße erreichen [13] ( Abb. 1). Das Aneurysma spurium trat oft in der Ellenbeuge nach einem unvorsichtigen Aderlass mit Verletzung der A. brachialis auf [9] ( $\triangleright$ Abb. 2). Beim Aneurysma cirsoideum handelt es sich um eine kongenitale Anomalie oft im Bereich des Kopfes, die im Laufe des Lebens mitunter bis zur Entstellung gewachsen ist [3] ( $\triangleright$ Abb. 3). Das Aneurysma varicosum wurde dadurch definiert, dass sich zwischen der betroffenen Arterie und der Vene 2 Aneurysma-Säcke befinden; in den ersten Sack fließt das Blut aus der Arterie hinein und aus dem zweiten Sack in die Vene ab ( $\triangleright$ Abb. 4). Diese Säcke fehlen beim Varix aneurysmaticus [1, 9, 18] ( Abb. 5a). Heute bezeichnen wir diese letztere Form als arteriovenöse Fistel oder arteriovenösen Shunt. Die Krankheitsgeschichte eines Soldaten des 2. Königlich Württembergischen Infanterie-Regiments, der sich den Varix aneurysmaticus während des Urlaubs zugezogen hat, führt uns in die damaligen Verhältnisse zurück.

\section{TYPEN VON ANEURYSMEN \\ $[1,9,18]$ \\ Aneurysma verum \\ Aneurysma spurium \\ Aneurysma racemosum (mixtum) \\ Aneurysma varicosum \\ Varix aneurysmaticus}

\section{Das Königlich Württembergi- sche Infanterie-Regiment}

Im Königreich Württemberg regierte König Wilhelm I (1781-1864) von 1816 bis1864 und unterhielt ein kleines Heer mit 13955 Soldaten und 18 Geschützen. Es bestand die Wehrpflicht für junge
Männer [12] (https://de.wikipedia.org/ wiki/W\%C3\%BCrttembergische_Armee). Anno 1832 kehrte der Soldat J. G. Schäfer aus Gemmrigheim, einem kleinen Dorf bei Ludwigsburg, zu seiner Einheit zurück und stellte sich beim Regimentsarzt Dr. Seeger wegen der Verletzung nach einer Wirtshausstreiterei vor. Hier, bei der Armee, begann erst einmal alles mit der disziplinarischen Strafe: „Der Patient mußte wegen des Streites, bei welchem ihm die Verletzung beigebracht worden war, einen vierzehntätigen Arrest zweiten Grades (also bei schmaler Kost) erstehen" [15].

\section{Krankheitsbericht des Regimentsarztes Dr. Seeger}

Über den Untersuchungsbefund schrieb Seeger: Ich fand einen robusten, wohlge-

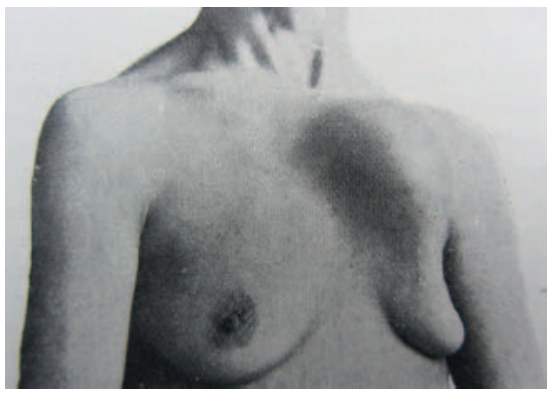

Abb. 1 Großes Aneurysma des Arcus Aortae [13].

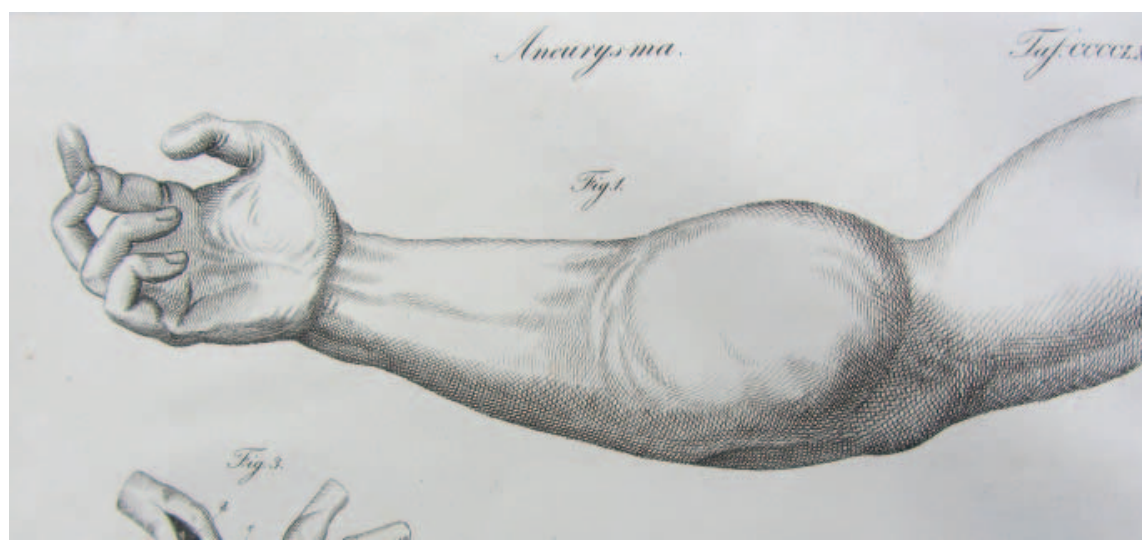

- Abb. 2 Aneurysma spurium. Arm, an welchem bei einem Aderlaß die Arterie angestochen war und eine Blutergießung in das umgebende Zellgewebe Statt gefunden hatte. Froriep Tafel CCCCLXXXIII, Fig. 1 [3]. wachsenen, gesund aussehenden Burschen von 23 Jahren, welcher früher nie an einer Krankheit gelitten hatte, dessen Eltern auch bisher immer gesund gewesen waren. Er litt an einer klopfenden Geschwulst am linken Oberschenkel. Hierzu heißt es, dass er am 27. März 1832 abends gegen 22 Uhr von einem anderen Bauernburschen, mit dem er am Vortag in Streit geraten war, durch

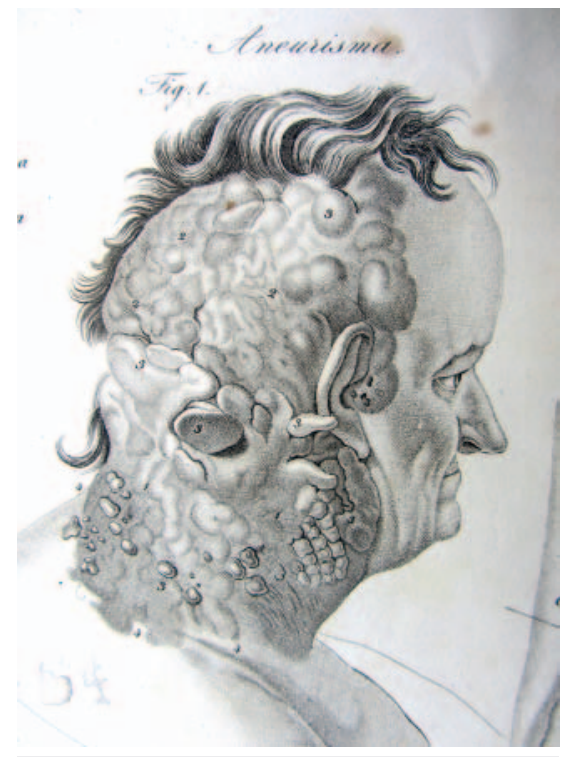

Abb. 3 Aneurysma verum cirsoideum bei einer 72-jährigen Frau aus der Salpètrière in Paris. Bei der Geburt bläulicher Fleck am Hinterkopf. Seit dem 6. Lebensjahr zunehmende Pulsationen. Froriep Tafel CCCLXVI, Fig. 1 [3]. 


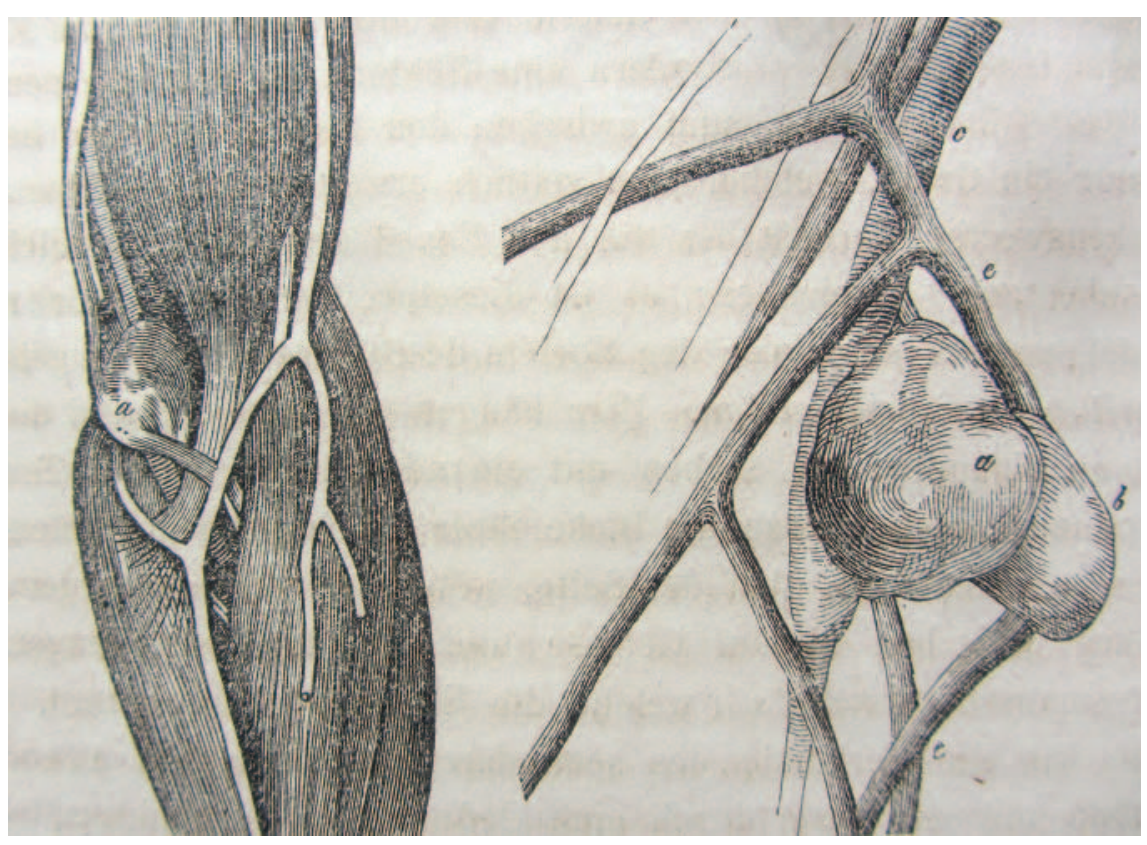

- Abb. 4 a, b Wenig entwickeltes Aneurysma varicosum. Entstehung durch Aderlass, wobei die $\mathrm{V}$. mediana basilica durchstochen und die darunterliegende Arterie angestochen waren. a Anatomisches Präparat. b Seitliche Ansicht in natürlicher Größe. Größerer Sack a der Arterie, kleinerer Sack b der Vene zugehörig [1].

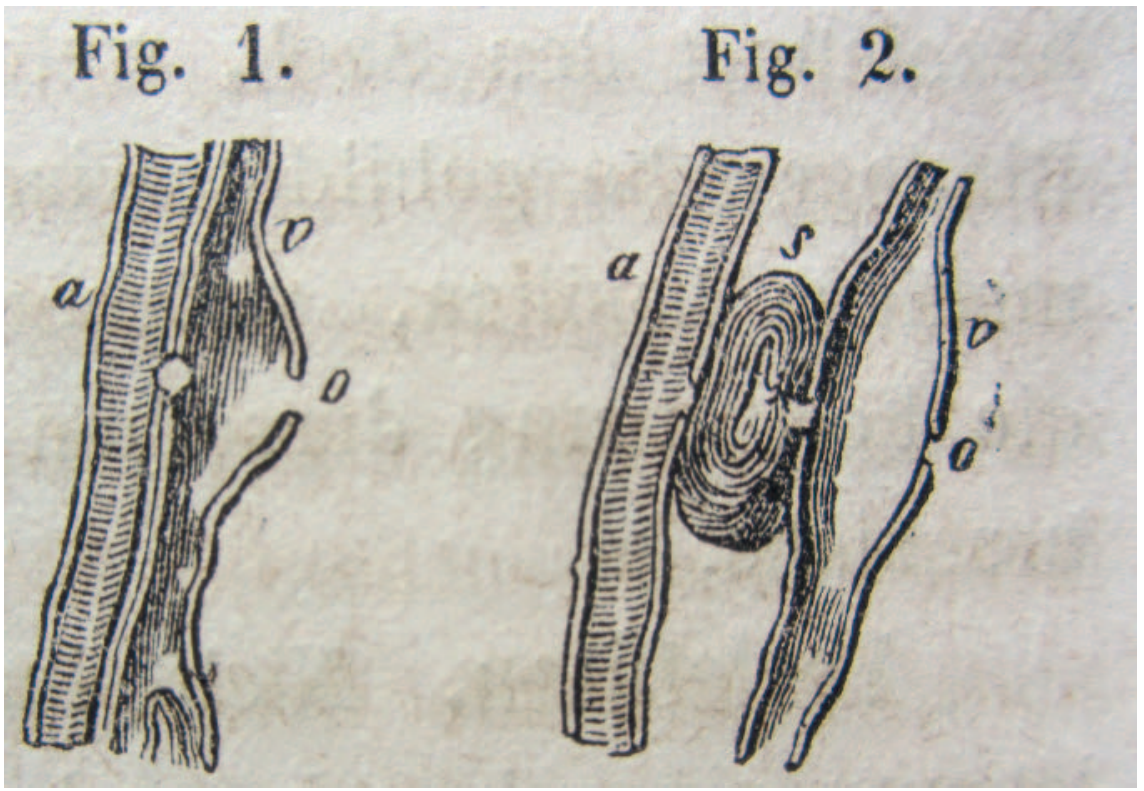

Abb. 5 a, b Varix aneurysmaticus und Aneurysma varicosum. a Gedachter Längsdurchschnitt durch Arterie (links) und Vene (rechts), dazwischen (o) die Verbindung zwischen den Gefäßen. b Venöses Aneurysma. Vene und Arterie sind durch den aneurysmatischen Sack verbunden [1].

ein Taschenmesser verletzt wurde. Die Klinge drang $5 \mathrm{~cm}$ unterhalb der Leiste in den Oberschenkel ein, und sofort spritzte arterielles Blut aus der Wunde. Schäfer konnte sich noch 300 Schritte bis vor die Tür des Dorfchirurgen schleppen, dann brach er zusammen. Vom Wundarzt wurde ein fester Verband angelegt, anschließend trug man ihn auf einem Lehnsessel nach Hause. Dort fiel er wieder in Ohnmacht. Die starke Blutung hielt an. Erst ein zweiter, fester Verband führte zum
Erfolg. Aber dadurch traten heftige, reißende Schmerzen im Unterschenkel auf. Am 6. Tag begann die Wunde zu eitern, dann heilte sie ab [15].

Nach 4 Wochen entdeckte der Chirurg an der Narbe eine haselnussgroße, bläuliche Geschwulst, die sichtbar pulsierte. In der Umgebung fühlte man ein eigenthümliches Schwirren, das dem Finger ein eigenthümliches Gefühl von Pelzigseyn und Prickeln wie von einem kleinen elektrischen Strom mittheilt. Auf die Geschwulst drückend fühlte ich deutlich eine der Narbe nicht in ganzer Größe entsprechende Oeffnung in der Arterie und den durch diese hindurchgehenden flachen Blutstrom. Mit dem aufgelegten Ohr und dem Stethoskop waren pulsierende Geräusche zu hören. Der Kranke fühlte das Schwirren bis in den Bauch hinein. Seine Gesichtsfarbe und besonders die Lippen ließen etwas Bläuliches nicht verkennen. Die ganze linke untere Extremität war etwas kühler anzufühlen.

Der Soldat verspürte keine Schmerzen und verrichtete seinen Dienst in gewohnter Weise. Der nach innen gerichtete Sack des Varix war in seinen Wandungen dicker und sehr deutlich geworden. Ein Jahr später hatte sich eine Vene in der Nähe etwas erweitert [15].

\section{Erklärung von Dr. Seeger}

Ich glaube: In einer unmittelbaren Communikation der Arterien- und Venenwunde hat sich die Öffnung zwischen beiden nicht geschlossen, in deren Folge das Blut unmittelbar von der Arterie in die Vene überströmt, und wobei sich letztere nach oben und unten varicös ausgedehnt hat; folglich in einem $>$ Varix aneurysmaticus< nach Hunter. Die nahe gelegene Vene, welche das ergossene Blut das in das Aneurysma aufnimmt, dehnt sich varicös aus [15].

\section{Therapeutische Überlegungen}

Aufgrund der Beobachtungen aus der Literatur müssten das Aneurysma spurium und das Aneurysma varicosum operiert werden, der Varix aneurysmaticus aber nur selten. Oft reicht hier die lokale Kompression aus. Nur in einem von Larrey sowie einem von Dupuytren beschriebenen Fall mussten die Gliedmaßen amputiert werden [15].

Dr. Seeger meinte, in unserem Falle wurden anfangs Heilungsversuche durch Com- 


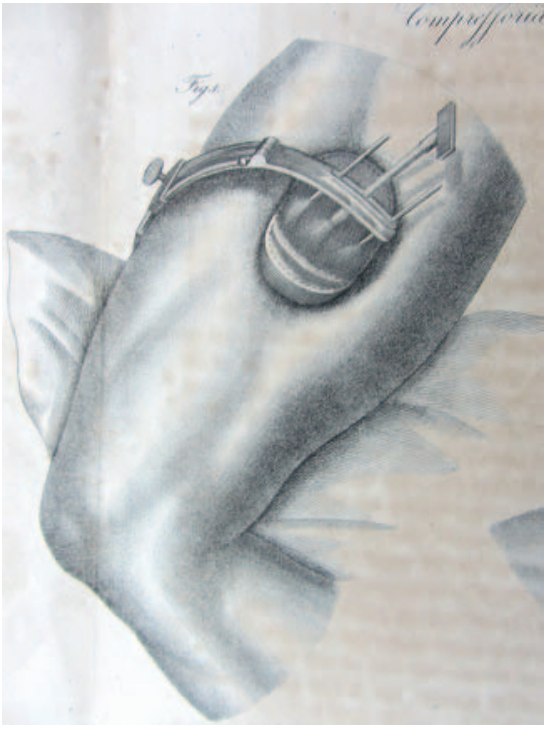

- Abb. 6 Tourniquet zur Kompression $\operatorname{der}$ A. femoralis von Dupuytren. Feste Andrückung $\operatorname{der}$ A. cruralis (A. femoralis) gegen den Körper des Schenkelbeins. Froriep Tafel CCCCVI, Fig. 1 [3]

pression und styptische Mittel gemacht, aber ohne Erfolg. Man rieth dem Patienten als Bauern und Weingärtner, nur leichtere Arbeiten zu verrichten, und sich jedes Excesses im Essen und Trinken u. s. w. zu enthalten. Seit einiger Zeit trägt er aber eine auf unser Anrathen verfertigte bruchbandartige Bandage mit starker Feder und großer Pelotte, welche ihren Druck unmittelbar auf die Geschwulst ausübt ( Abb. 6, 7). Hierdurch wurden ihm die lästigen Gefühle sehr gemildert, und er verrichtet jetzt selbst schwere Arbeiten ohne Beschwerden [3, 15].

\section{Weitere Kasuistiken}

In der Literatur stammten die ersten beiden Beschreibungen des Varix aneurysmaticus von William Hunter (1718-1783, Chirurg in London). In seinem Referat am 24. August 1761 hat er die Kasuistiken auf der Tagung der Society of Physians in London vorgetragen. Die Publikation erfolgte anno 1763 in kurzer Form [8] und dann im folgenden Jahr 1764 mit 2 ausführlichen Fällen in der Zeitschrift Medical Observations and Inquiries [9]. Heute ruft es größte Hochachtung hervor, mit welcher Genauigkeit und Weitsicht der Forscher damals schon aufgrund der klinischen Befunde allein dem Krankheitsbild auf die Spur kam.

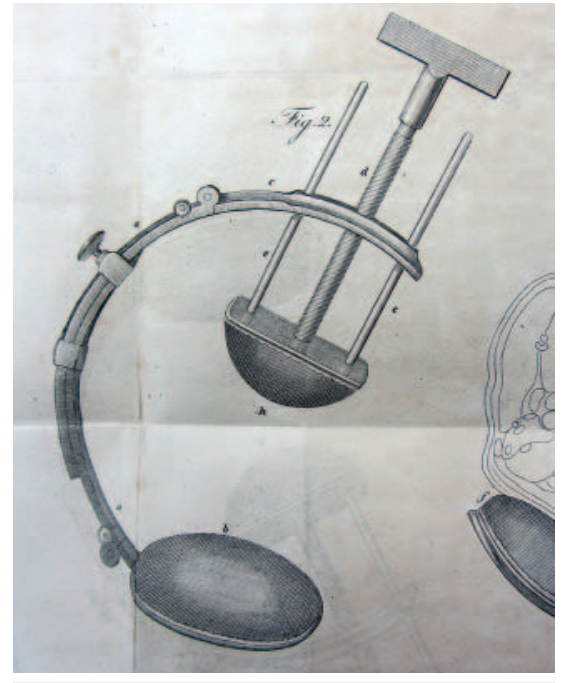

- Abb. 7 Instrument zur Kompression. Vermeidung einer Umschnürung des Beins. Froriep Tafel CCCCVI, Fig. 2 [3].

\section{William Hunters Fall anno 1761}

Der Patient Thomas Brookhouse Chefhire (CASE II) wurde vor 5 Jahren zur Ader gelassen. Er hatte schon bei der Prozedur selbst das Gefühl, dass die Lanzette zu tief eingestochen wurde. Zunächst ergab sich nur ein starker Bluterguss am Arm. Dann bildete sich eine Erweiterung der Gefäße aus. Sowohl die Arterie als auch besonders die Venen waren bis zur Achsel sehr stark angeschwollen und geschlängelt. Durch das Auflegen des Ohres ließen sich laute Strömungsgeräusche hören. Bei Kompression des Arms unterhalb der Ellenbeuge nahmen alle Strömungen bis zur Achselhöhle zu, bei alleiniger Kompression der Arterie am Oberarm ging die venöse Füllung zurück. I shall not take up the reader's time with enquiring by what name the disorder should be called, whether Aneurysm or varix, or both, or neither, as it is obvious that every surgeon would naturally reckon it a species of Aneurysm [9]. Hunter führte eine Kompressionstherapie durch.

\section{Hodsons Fall anno 1817}

Joseph Hodson (1788-1869, Chirurg in Birmingham) teilte die Kasuistik eines 17-jährigen Schmiede-Lehrlings mit. Durch den Unfall mit einer fast rothglühenden Eisenstange kam es am Oberschenkel zu einer heftigen Blutung. Ein Geselle drückte die Wunde mit den Fingern ab, bis ein Wundarzt die Behandlung mit
Zirkelverbänden übernommen hatte. Nach 8 Wochen war die Wunde verheilt. Zehn Wochen nach dem Vorfall erschien eine purpurfarbene Geschwulst von drei Zoll $(7,5 \mathrm{~cm})$ im Durchmesser unmittelbar auf der Femoralarterie, vier Zoll unterhalb dem Poupart'schen Bande (Leistenband). Sie besaß eine dem Puls am Handgelenke entsprechende Pulsation, und diese ward von einer eigenen zitternden Bewegung begleitet; mit der Bewegung aber verband sich ein summendes oder vielmehr zischendes Geräusch, so gleich dem von Hunter beschriebenen, daß man gar nicht zweifeln durfte, es sei die Eisenspitze durch die Femoralarterie in die Vene gedrungen und habe eine aneurysmatische Venengeschwulst hervorgebracht. Der Kranke wurde mit einer über den ganzen Schenkel geschnürten Binde und einer festen Compresse, die von Kork mit Leder überzogen auf die Geschwulst anzulegen war, nach einem Monat aus dem Krankenhaus entlassen. Er konnte ziemlich gut gehen, die Geschwulst war etwas kleiner und das Klopfen weniger heftig [6, 7].

\section{Schösslins Fall anno 1823}

Über den Arzt oder Wundarzt Schösslin ist in der Literatur nur die Beschreibung seiner Kasuistik in Köstrin, einem Dorf heute in Polen, bekannt. Es handelte sich um eine 65-jährige Frau, Mutter von 12 Kindern, die vor 55 Jahren eine Quetschung an der Handwurzel erlitten hatte. Bemerkenswerth ist die widernatürliche große Erweiterung der sämmtlichen Stämme der Arterien des kranken Arms. Schon über dem Schlüsselknochen am Stamme der Subclaviararterie beginnt sie in der Stärke eines Mannesdaumens, und setzt sich von da fort bis zur aneurysmatischen Venengeschwulst. Bei körperlicher Belastung entstand ein starkes Pochen des Herzens und der Armgeschwülste, Während der Schwangerschaft zeigte sich eine besondere Art von Orgasmus und führte zu Blutungen in das benachbarte Zellgewebe. Diese Erscheinung trat jedesmal mit der 20. Woche der Schwangerschaft ein und endete 4 Wochen vor der Niederkunft. Die Therapie erfolgte mit leichtem Kompressionsverband [5, 14].

\section{Stromeyers Fall anno 1841}

Georg Friedrich Louis Stromeyer (18041876, Chirurg in Hannover, München, Freiburg und Kiel) gehörte zu den genialen 
Chirurgen des 19. Jahrhunderts [17]. Seine Erfahrungen mit dem Fall eines Varix aneurysmaticus wurden wiederholt publiziert $[2,4,5,16]$. Eine Abbildung ist jedoch nicht bekannt. Es handelte sich um einen jungen Arzt, dem bei einem Duelle die linke Temporalarterie, die große Gesichtsvenen und der Gesichtsnerv durchschlagen wurden. Die Lähmung des Facialis hatte sich allmählich bis auf geringe Spuren verloren, ein Aneurysma war geblieben, welches, durch Communication von Arterie und Vene entstanden, ein sehr entstellendes Hervortreten der Frontalvenen an der Stirn und der übrigen Kopfvenen veranlasst hatte. Der junge Mann sah aus wie eine Medusa, die große Gesichtsvene war dicht unter dem Ohr verschlossen. Prof. Maximilian Joseph von Chelius (17941876) in Heidelberg hatte bereits die Carotis ohne Erfolg unterbunden [2, 4, 16].

Stromeyer führte eine komplizierte Operation durch. Er hat sowohl die kommunizierende Arterie nach oben als auch die Vene nach unten ligieren können. Der junge Mann wurde durch die Operation so verschönert, daß man ihn kaum wieder erkennen konnte $[5,16]$.

\section{Schlussbetrachtung}

Der Varix aneurysmaticus und das Aneurysma varicosum, wie sie von Hunter und später von Bardeleben durch die Ausbildung eines Sacks zwischen der arteriovenösen Kommunikation differenziert wurden, kamen als Folge einer Fremdeinwirkung in früheren Jahrhunderten sicherlich nicht allzu oft vor. In der Regel handelte es sich um Stichverletzungen an den Extremitäten, wo Schlagader und Vene dicht beieinander liegen. In der Ellenbeuge kamen sie durch den Aderlass und am Oberschenkel durch Stich- und Schusswunden zustande. Aber jeder Chirurg dürfte in seinem Leben nur selten damit konfrontiert worden sein.

Die erste Beschreibung des Varix aneurymaticus geht anno 1763 auf William Hunter zurück [8, 9]. Heute erstaunt es, mit welcher Genauigkeit der berühmte englische Arzt die einzelnen Symptome des Krankheitsbildes beobachtete und so der Pathophysiologie auf die Spur kam. Auf seine Publikation hin folgten mehrere Zuschriften an die Society of Physicians in London mit entsprechenden Krankheitsfällen anderer Kollegen [10, 11]. Als glücklichen Umstand darf man bezeichnen, dass Hunters Arbeit eine so weite internationale Verbreitung gefunden hat, sie wurde immer wieder zitiert.

Die logische Operation bestand in der Ausschaltung der arteriovenösen Fistel. Das ist auch heute noch ein anspruchsvoller gefäßchirurgischer Eingriff. Die proximale und distale Unterbindung der Arterie ist Stromeyer erstmals an der A. temporalis gelungen [16]. Seinerzeit kam man in der Regel mit der Kompressionstherapie aus. Dazu wurde von dem Chirurgen ein spezielles Kompressorium angefertigt, das den arteriovenösen Shunt möglichst genau treffen und den Blutstrom reduzieren sollte. Auf diese Weise konnten die meisten Patienten mit dem Varix aneurysmaticus oder dem Aneurysma varicosum ihr Leben meistern

Der Schmerz, der uns zugefügt wird, ist nicht die schwerste Last des Lebens. Viel schwerer legt sich eines Tages auf unsere Schultern der Schmerz, den wir den anderen zugefügt haben. Hermann Bang (1857-1912, dänischer Schriftsteller)

Interessenkonflikt

Die Autorinnen/Autoren geben an, dass kein Interessenkonflikt besteht.

\section{Autorinnen/Autoren}

Wolfgang Hach

Korrespondenzadresse

Prof. Dr. Wolfgang Hach

Tacitusstr. 66

60439 Frankfurt am Main

Tel.: ++ 49/69/573271

hach.frankfurt@gmx.de

\section{Literatur}

[1] Bardeleben A. Lehrbuch der Chirurgie und Operationslehre. 2. Auflage. Bd II Berlin: Reimer; 1859: 87

[2] Burckhardt C. Ueber der Varix aneurysmaticus. Inaugural-Dissertation. Stuttgart: Ebner und Seubert. 1843

[3] https://books.google.de/books?id=WBdPAAAA cAAJ\&printsec $=$ frontcover $\&$ hl $=$ de $\&$ source $=g$ bs ge_summary_r\&cad $=0 \# v=$ onepage\& $q \&=$ false; am 2.5.2020
[4] von Froriep LF. Chirurgische Kupfertafeln. Bd III Weimar: Gr. Herzogl. Sächs. priv. Landes-Industrie-Comptoir; 1823

[5] Giessen GE. Geschichte eines Aneurysma varicosum, nebst einer Einleitung über das Aneurysma varicosum. Inaugural-Dissertation. München;, im Januar 1842

[6] https://books.google.de/books?id=_wNPAA AAcAAJ\&pg $=P P 1 \& l p g=P P 1 \& d q=D r .+$ G.E. + Giessen+Aneurysma+varicosum\&source= bl\&ots=QilAlpai9f\&sig=ACfU3U1 toXpfjo2qso wad7pTt99|CumtQQ\&hl=de\&sa=X\&ved=2ah UKEwjX0pGTq5XoAhUIGsAKHYH5DzQQ6AE wAnoECAYQAQ\#v=onepage \&q=Dr.\%20G.E. \%20Giessen\%20Aneurysma\%20varico sum\&f=false; am 2.5.2020

[7] Hach W. Georg Friedrich Louis Stromeyer (1804-1876) und die Operation eines Varix aneurysmaticus. Gefäßchirurgie. Im Druck

[8] Hodgson JH. Von den Krankheiten der Arterien und Venen mit besonderer Rücksicht auf Entwicklung und Behandlung der Aneurysmen und Arterienwunden. Aus dem Englischen übersetzt von Kreysig. Hannover: Gebrüder Hahn. 1817: S. 514

[9] Hodgson JH. In Hirsch A. Biographisches Lexikon der hervorragenden Aerzte aller Zeiten und Völker. Wien und Leipzig: Urban und Schwarzenberg; 1887

[10] Hunter W. The history of an Aneurysm of the Aorta, with some Remarks on Aneurysms in general. Medical Observations and Inquiries 1763; 1: 323-357 (hier S. 340)

[11] https://books.google.de/books?id=kvc_AA AAcAAJ\&pg=PA8\&dq=Medical+observations. +and+inquiries\&hl=de\&sa=X\&ved=0ahU KEwj2raLArJ_oAhUHmYsKHQj9CgIQ6AEIK DAA\#v $=$ onepage $\& q=$ Medical\%20observati ons.\%20and\%20inquiries\&f=false; am 2.5.2020

[12] Hunter W. Further Observations upon a particular species of Aneurysm. Medical Observations and Inquiries 1764; 2: 390414

[13] https://books.google.de/books?id=n_c_AA AAcAAJ\&pg=PA75\&dq=Medical+obs. + and + inquiries\&hl=de\&sa=X\&ved=0ahUKEwjA 7qSoop_oAhUSxosKHR81CysQ6AEIK

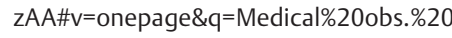
and\%20inquiries\&f=false; am 2.5.2020

[14] Hunter W. Letter to Hunter. Further Observations upon a particular species of Aneurysm. Medical Observations and Inquiries 1764; 2: 360-364

[15] Hunter W. Postscrift to the proceeding case of the varicose Aneurysm. Medical Observations and Inquiries 1771; 4: 385-387

[16] https://books.google.de/books?id=u_c_AAAA cAAJ\&printsec $=$ frontcover $\&$ hl $=$ de $\&$ sour ce $=$ gbs_ge_summary_r\&cad $=0 \# v=$ onepage $\&$ q\&f=false; am 2.5.2020

[17] Königreich Württemberg. https://de. wikipedia.org/wiki/W\%C3\% BCrttembergische_Armee; am 2.5.2020

[18] Romberg E. Krankheiten des Herzens und der Blutgefäße. 3. Auflage Stuttgart: Enke; 1921: 644 
[19] Schösslin Dr. Aneurysmatische Venengeschwulst. Frorieps Notizen aus dem Gebiete der Natur- und Heilkunde 1822; 3: 137-142

[20] Seeger H. Ueber den Varix aneurysmaticus nebst einem hieher gehörigen Fall. Medicinische Annalen 1 1825; H2: 268-295

[21] https://books.google.de/books?id=JaT_ BTzettoC\&printsec $=$ frontcover $\& \mathrm{hl}=\mathrm{de} \# \mathrm{v}=$ onepage\&q\&f=false; am 2.5.2020

[22] Stromeyer GFL. Erinnerungen eines deutschen Arztes. 2 Bd Hannover: Rümpler; 1875

[23] Stromeyer GFL. In Hirsch A. Biographisches Lexikon der hervorragenden Aerzte aller Zeiten und Völker. Wien und Leipzig: Urban und Schwarzenberg; 1887
[24] Travers BT. Von dem aneurysmatischen Varix und dem Aneurysma varicosum. In: Die chirurgische Praxis der bewährtesten Wundärz te unserer Zeit systematisch dargestellt. Bd 3/1. 113-115 Berlin: Voss'sche Buchhandlung; 1841

[25] https://books.google.de/books?id=rEg_AA AAcAAJ\&pg=PA113\&dq=Travers, +Breschet + +Von+dem+aneurysmatischen+Varix+und+ dem+Aneurysma+varicosum. \&hl=de\&sa= X\&ved=0ahUKEwiQmYK7gpPpAhWR7KYK HebMDZ8Q6AEIJzAA\#v=onepage \&q=Travers \%2C\%20Breschet\%20.\%20Von\%20dem\% 20aneurysmatischen\%20Varix\%20und\%
20dem\%20Aneurysma\%20varicosum. $\& \mathrm{f}=$ false; am 2.5.2020

Bibliografie

Phlebologie 2021; 50: 100-104

Online-Publikation: 9.10.2020

DOI $10.1055 / a-1175-4973$

ISSN 0939-978X

(c) 2020. Thieme. All rights reserved.

Georg Thieme Verlag KG, Rüdigerstraße 14, 70469 Stuttgart, Germany 